\title{
Sex Ratio Conflict and Worker Production in Eusocial Hymenoptera
}

\author{
Max Reuter ${ }^{*}$ and Laurent Keller
}

Institute of Ecology, University of Lausanne, 1015 Lausanne, Switzerland

Submitted July 13, 2000; Accepted March 27, 2001

ABstract: The best known of the conflicts occurring in eusocial Hymenoptera is queen-worker conflict over sex ratio. So far, sex ratio theory has mostly focused on optimal investment in the production of male versus female sexuals, neglecting the investment in workers. Increased investment in workers decreases immediate sexual productivity but increases expected future colony productivity. Thus, an important issue is to determine the queen's and workers' optimal investment in each of the three castes (workers, female sexuals, and male sexuals), taking into account a possible trade-off between production of female sexuals and workers (both castes developing from diploid female eggs). Here, we construct a simple and general kin selection model that allows us to calculate the evolutionarily stable investments in the three castes, while varying the identity of the party controlling resource allocation (relative investment in workers, female sexuals, and male sexuals). Our model shows that queens and workers favor the investment in workers that maximizes lifetime colony productivity of sexual males and females, whatever the colony kin structure. However, worker production is predicted to be at this optimum only if one of the two parties has complete control over resource allocation, a situation that is evolutionarily unstable because it strongly selects the other party to manipulate sex allocation in its favor. Queens are selected to force workers to raise all the males by limiting the number of eggs they lay, whereas workers should respond to egg limitation by raising a greater proportion of the female eggs into sexual females rather than workers as a means to attain a more female-biased sex allocation. This tug-of-war between queens and workers leads to a stable equilibrium where sex allocation is between the queen and worker optima and the investment in workers is below both parties' optimum. Our model further shows that, under most conditions, female larvae are in strong conflict with queens and workers over their developmental fate because they value their own reproduction more than that of siblings. With the help of our model, we also investigate how variation in queen number and number of matings per queen affect the level of conflict between queens, workers, and larvae and ultimately the allocation of resource in the three

\footnotetext{
* E-mail: max.reuter@ie-zea.unil.ch.
}

Am. Nat. 2001. Vol. 158, pp. 166-177. (c) 2001 by The University of Chicago. 0003-0147/2001/15802-0006\$03.00. All rights reserved. castes. Finally, we make predictions that allow us to test which party is in control of sex allocation and caste determination.

Keywords: Hymenoptera, colony growth, kin selection, conflict, model.

One of the major trade-offs in an organism's life history is that between investment in reproduction versus body growth (Stearns 1992). Spending resources on immediate reproduction provides direct fitness gain but decreases somatic growth and expected future reproduction. The evolutionarily optimal strategy is continued growth until the fitness gain as a result of increased size is exactly counterbalanced by the fitness obtained by immediate reproduction. This principle can be used to predict investment in colony growth in social insects with morphological castes, in which workers are the equivalent of somatic tissue and new queens (gynes) and males constitute the reproductive investment. However, in contrast to unitary organisms that consist of clonal cells $(r=1)$, colonies of social insects contain individuals whose relatedness is lower than 1 $(r<1)$. This genetic heterogeneity results in potential genetic conflicts over resource allocation and reproductive decisions (Hamilton 1964; Pamilo 1991; Ratnieks and Reeve 1992; Keller and Chapuisat 1999; Keller and Reeve 1999). Genetic conflicts are of particular importance in eusocial Hymenoptera (ants, bees, and wasps), whereby the haplodiploid sex-determination system creates relatedness asymmetries among family members. Because workers are more closely related to their sisters than to their brothers, they should favor a female-biased sex allocation (here defined as the relative investment in gynes and males). In contrast, queens are equally related to their sons and daughters and favor an even resource investment in both sexes. Thus, queen-worker conflict over sex allocation arises.

Sex ratio conflict has been extensively investigated both theoretically and empirically (e.g., Trivers and Hare 1976; Charnov 1978; Nonacs 1986; Boomsma and Grafen 1990, 1991; Pamilo 1991; Sundström 1994; Bourke and Franks 1995; Crozier and Pamilo 1996; Chapuisat and Keller 1999). In contrast, little attention has been paid to the 
question of how conflict among colony members extends from resource allocation to colony growth and maintenance (worker production) versus reproduction (production of males and gynes). The different authors who have asked this question have reached different conclusions. Pamilo (1991) suggested that conflict over colony growth arises between queens and workers, with workers preferring a relatively greater investment in gynes than workers as compared to the queen. However, Bourke and Chan (1999) suggested that conflict between queens and workers should be absent if the relative investment in worker and sexual production does not affect a colony's sex allocation (the proportional investment in gynes vs. males). That is, both queens and workers should favor the investment in workers that ultimately maximizes the lifetime colony production of reproductive individuals. Finally, yet another conclusion was reached by Bourke and Ratnieks (1999) when they took into account the process of caste determination, whereby female larvae irreversibly develop into workers or queens that are morphologically and physiologically specialized for the task that they will perform. The authors found that conflict between queens and workers arose over the developmental pathway of individual female larvae, with workers preferring a higher proportion of female larvae to develop into gynes than the queen. Bourke and Ratnieks's (1999) analysis also included the interests of the female brood itself. They found that female larvae are in conflict with queens and workers under a wide range of conditions. Because female larvae are more closely related to themselves than to the sexual females they would raise as workers, they frequently benefit by becoming sexual females themselves under conditions where queens and workers would benefit from increasing the size of the colony's workforce.

In this article, we reconsider the problem of potential conflict over resource allocation in the production of workers, males, and gynes. We construct a simple and general kin-selection model that allows us to investigate the simultaneous evolution of investment in workers and sex allocation. This is a significant step toward a better understanding of optimal reproductive allocation and sex ratio conflict because previous models dealt either with sex allocation, neglecting investment in colony growth, or with optimal investment in colony growth in the situation where sex allocation is fixed. Moreover, in our analyses, we explicitly consider the power that queens and workers have to manipulate resource allocation and the selection pressures that act on queens, workers, and larvae to encourage manipulation.

Our model shows that queen-worker conflict occurs over sex allocation but not colony growth. The evolutionarily stable investment in workers is the same for both parties when one of them has complete control over re- source allocation (i.e., the relative investment in workers, gynes, and males). Complete control by one party is, however, evolutionarily unstable because it strongly selects the other party to manipulate sex allocation in its favor. When no party can monopolize control over resource allocation, the investment in worker production is predicted to be lower than the two parties' optimum. This is because the queen's and workers' attempts to bias sex allocation in their favor are at the detriment of worker production. Our model also confirms the occurrence of conflict between adult colony members and female larvae over their developmental fate. Under most conditions, individual female larvae benefit if they escape adult control of caste determination and develop into gynes. Finally, our model sheds light on the reasons why previous authors reached different conclusions about whether queens and workers are in conflict over the relative amount of resources that should be allocated to worker production.

\section{The Model}

In our model, we consider a colony with one or several queens (mated with a variable number of males) and sterile workers. Males and gynes are assumed to disperse so that inbreeding, local mate competition (Hamilton 1967), or local resource competition (Clark 1978) do not occur. Workers, gynes, and males are assumed to be equally costly to produce. Therefore, the proportional investment in one class of individuals corresponds to the number of individuals of this class relative to the total number of individuals produced. We will thus use the terms "sex ratio" and "sex allocation" interchangeably throughout the article. In the colony, investment in workers, gynes, and males is determined by two parameters. The first, $f$, describes the proportion of resources that are allocated to the production of females (i.e., workers and gynes, hereafter referred to as "proportional investment in females"). Correspondingly, the quantity $(1-f)$ is the proportion of resources invested in the production of males. The second parameter determining resource allocation is $w$, the proportion of the allocation to females that is devoted to the production of workers. The remainder $(1-w)$ goes into the production of gynes. Because of the equal cost of gynes and workers, the parameter $w$ therefore describes the proportion of females that will develop into workers. In sum, the colony invests a proportion $f w$ of resources in workers, a proportion $f(1-w)$ in gynes, and $(1-f)$ in males. The population means of the proportional investments are given by capital letters, that is, $F W$ into workers, $F(1-W)$ into gynes, and $(1-F)$ into males. For the sake of simplicity, our model does not consider any temporal variation of investment that might occur in the course of colony ontogeny. It predicts the overall invest- 
ment in workers, gynes, and males over the whole colony life.

Because per-worker productivity declines with the number of workers per colony in eusocial Hymenoptera (Brian 1956; Tschinkel 1993; Sundström 1995), overall colony productivity (biomass of workers, gynes, and males produced) is assumed to increase with colony size, according to the following diminishing return function: $b(f, w)=$ $1-(1-f w)^{2}$. Under this function, productivity varies between 0 , when the relative investment in workers is 0 , and 1 (maximum productivity), when all resources are allocated to worker production (fig. 1, solid line). The productivity in terms of sexual biomass (gynes and males) is given by $b(f, w)(1-f w)$ (fig. 1 , dotted line). (Note that choosing another function with diminishing returns does not affect the qualitative results of our model.)

The inclusive fitness $V_{X}$ of a colony member $X$ depends on the biomass of males and gynes produced, the regression relatedness of females $\left(g_{F X}\right)$ and males $\left(g_{M X}\right)$ to $X$, the reproductive values of females $\left(\nu_{F}=1\right)$ and males $\left(\nu_{M}=0.5\right)$, and the mating success of female reproductives $(1 /[F(1-W)])$ and males $(1 /[1-F])$ (Crozier and Pamilo 1996). Taking into account these variables, the fitness of individual $X$ is given as

$$
V_{X}=b(f, w)\left[\frac{f(1-w) g_{f X} \nu_{f}}{F(1-W)}+\frac{(1-f) g_{m X} \nu_{m}}{(1-F)}\right]
$$

The fitness function (1) allows us to calculate the evolutionarily stable (ES) values for the proportional investment in females $f$ and the proportion of females developing into workers $w$ in function of who in the colony has control of these two variables and what the kin structure of the colony is.

The question of who controls the two parameters ( $f$ and $w)$ that determine resource allocation is important because queens and workers might differ in their optimal values. The queen can produce males by laying unfertilized haploid eggs and females by producing fertilized diploid eggs. She is therefore a priori in control of proportional investment in females. However, workers may alter the sex allocation by selectively eliminating brood of one sex (most likely males, as has been demonstrated in the ant Formica exsecta; Sundström et al. 1996; Chapuisat et al. 1997) to raise more individuals of the other sex. In this way, workers can gain control over proportional investment in females.

There has been considerable discussion about who (the queen, the workers, or the female larvae) is in control of caste determination and therefore able to regulate the proportion of females that develop into workers versus gynes. Female development had long been assumed to be manipulated by queen-produced pheromones (Brian 1980;

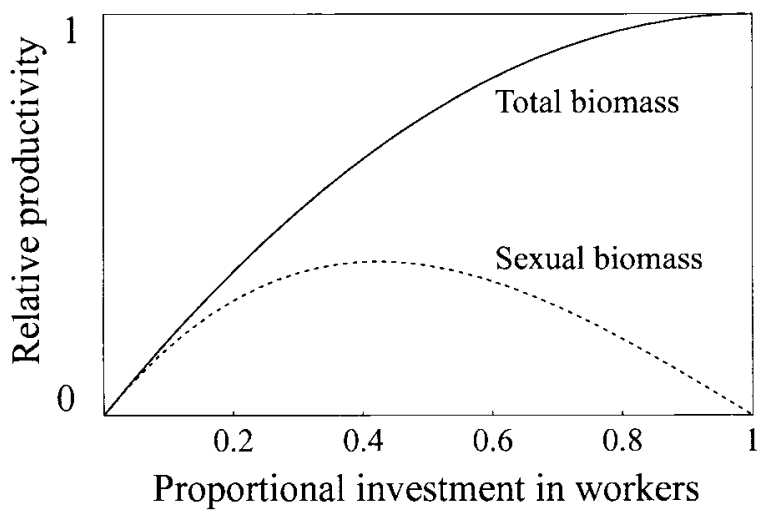

Figure 1: Relative productivity of a colony as a function of proportional investment in workers. The solid line shows the total productivity (workers and sexuals), the dotted line, the productivity of sexuals only.

Fletcher and Ross 1985; Hölldobler and Bartz 1985). However, this view has been challenged on theoretical grounds, and there is indeed no empirical data in favor of pheromonal control, whereby queens are able to chemically force female larvae to develop into workers rather than queens (Keller and Nonacs 1993). On the contrary, there is increasing evidence that queen pheromones act as honest signals of queen fecundity to which other colony members respond by altering reproductive decisions (e.g., Pettis et al. 1997; Ortius and Heinze 1999). An alternative way for queens to influence caste determination is by producing different types of eggs, which has been documented in two species of ants. In Pheidole pallidula, queens produce diploid eggs that vary in their content of juvenile hormone and develop either into workers or gynes (Passera 1980; Wheeler 1986). Similarly, Formica polyctena queens can apparently bias caste determination by varying egg size and the amount of RNA and other biochemical substances in the eggs (Wheeler 1986, and references therein). Since it is yet unclear who is controlling caste determination and because there might be interspecific variation, we will consider the three possible scenarios of caste determination, with the developmental fate of larvae being either under the control of the queen, the workers, or the larvae themselves.

In our model, we will first consider the case where either the queens or the workers have complete control over $f$ and $w$, that is, the proportional investment in females and the proportion of females raised as workers. Complete queen control may occur when queens limit the number of eggs produced, thus making eggs a limiting resource, thereby forcing workers to raise all the brood present in the colony (Rosenheim et al. 1996), and when queens control caste determination, which may be the case in 
species with blastogenic caste determination (i.e., when maternal effects determine the developmental fate of female brood; Passera 1980; Pamilo 1982; Wheeler 1986; Helms 1999). Alternatively, complete worker control may occur when the number of female eggs is not limited, when workers are able to assess the sex of eggs or young larvae and eliminate males without any cost and thereby alter the queen-produced primary sex ratio, and when workers control caste determination, for example, through differential feeding of the larvae.

Next we will consider the case of mixed queen and worker control over resource allocation. Although singleparty control is possible under a restricted set of conditions (see above), queens and workers most likely share control over investment in the majority of species (Trivers and Hare 1976; Bourke and Franks 1995). This is because, when sex allocation is at one party's optimum, the other party is under strong selection to manipulate sex allocation to its own advantage. We will consider the most likely situation of mixed control (queens control the proportional investment into females [workers + gynes] by limiting the number of eggs, whereas workers control caste determination, i.e., the proportion of females developing into workers vs. the proportion developing into gynes). Note that, in this case, worker fitness will be principally limited by the number of female eggs available and not so much the overall number of eggs. Active egg limitation by the queen is therefore equivalent to a limitation of female eggs only, in which case workers raise all female eggs and invest the remaining resources in the rearing of as many male eggs as possible.

\section{Single-Party Control}

The ES investment pattern of a single party in control of resource allocation ( $f$ and $w$ ) can be found by substituting the corresponding values of relatedness for that party in equation (1) and maximizing the function simultaneously for $f$ and $w$ at $w=W=w^{*}$ and $f=F=f^{*}$. For queen control under monogyny (single-queen colonies) and monandry (single-queen mating), we substitute the relatedness values $g_{f}=0.5$ and $g_{m}=1$. The ES solution is $\left(f_{\mathrm{Q}}^{*}=0.71, w_{\mathrm{Q}}^{*}=0.59\right)$, meaning that a proportion of 0.71 of the resources is invested in females, of which a proportion of 0.59 goes into workers. The resulting allocation pattern is 0.42 of the resources invested in workers, 0.29 in gynes, and 0.29 in males. The investment in males and gynes is even, as predicted from sex ratio theory when queens control colony sex allocation (Trivers and Hare 1976), and the allocation to workers is the one that maximizes sexual production (see fig. 1). The numerical value of the relative investment in workers has of course no biological meaning as it critically depends on the function $b(f, w)$ that was chosen. A different ES investment in workers would be found if one would use a different function, but the important point is that it would always be the one that maximizes the overall productivity of males and gynes.

The ES values of $f$ and $w$ under complete worker control of resource allocation can be obtained in the same manner by substituting $g_{f}=0.75$ and $g_{m}=0.5$ in equation (1) and maximizing for $f$ and $w$. The values obtained are $\left(f_{W}^{*}=0.86, w_{W}^{*}=0.49\right)$, which corresponds to a proportional investment of 0.42 in workers, 0.44 in gynes, and 0.14 in males. The optimal investment in workers is the same as for queens, and it is the value that maximizes the colony's overall production of gynes and males. However, contrary to queens, workers favor a three times higher investment in gynes than in males, as predicted by sex ratio theory (Trivers and Hare 1976).

The more complex kin structures that result from polyandry (multiple mating by queens) or polygyny (multiple reproductive queens per colony) affect relatedness asymmetries between colony members and thus the workers' optimal sex allocation. However, under both complete queen control and complete worker control, the ES investment in workers is not influenced by the level of polyandry or polygyny because both parties benefit from maximizing overall colony productivity of gynes.

In summary, complete control of resource allocation by either queens or workers leads to each party favoring the relative investment in worker production that maximizes overall productivity of reproductives, and this is true whatever the kin structure of the colony. In contrast, queens and workers diverge in the optimal investment in males and gynes, as expected from sex ratio theory. Consequently, queen-worker conflict arises over the allocation of resources to male and female reproductives but not over worker production.

\section{Mixed Control}

When colony and population sex allocation are at one party's optimum, this exerts a selective pressure on the other party to bias sex allocation to its own advantage. Our model allows us to determine the strength and direction of this selective pressure by calculating the selection gradient, which is the coefficient of a regression of fitness on phenotype (Lande and Arnold 1983). The selection gradient is equivalent to the derivative of the fitness function with respect to phenotype. We can thus quantify the selection acting on queens and workers by calculating the partial derivatives of their inclusive fitnesses with respect to $f$ and $w$.

Consider first the case of complete worker control under monogyny and monandry. The predicted proportional investment in workers is 0.42 , and the sex allocation is female 
biased by $3: 1$. The gradient of selection on queens to alter the proportional investment in females is given by the partial derivative of equation (1) with respect to $f$. Substituting the values $g_{f}=0.5, g_{m}=1, F=0.86$, and $w=W=0.49$ gives a partial derivative of $\beta_{f}=$ $\delta V_{Q} / \delta f=-1.35$. This negative value indicates that complete worker control of resource allocation selects queens to decrease the colony's proportional investment in females, which can be achieved by producing a more malebiased primary sex ratio and limiting the number of eggs laid.

Alternatively, in the case of complete queen control of caste allocation, the proportional investment in workers is still 0.42 , but the sex allocation is now even. The gradient of selection acting on workers can be obtained by the partial derivative of worker fitness with respect to $w$ at $f=F=0.71, W=0.59$, and substituting the workers' relatedness to males and gynes $\left(g_{f}=0.75, g_{m}=0.5\right)$, which gives $\beta_{w}=\delta V_{w} / \delta w=-0.41$. This negative value indicates that workers benefit from directing a greater proportion of the female brood toward gyne development even though this decreases overall colony productivity.

In summary, neither complete queen control nor complete worker control of resource allocation are stable evolutionary equilibria. Both situations lead to strong selection on the party not in control of resource allocation to manipulate investment to its own favor. If manipulation is possible, mixed control of caste allocation will arise and queen and worker strategies of manipulation and countermanipulation will evolve in response to each other. A stable equilibrium will be reached when the queen cannot increase her inclusive fitness by altering the proportional investment in females via the primary sex ratio produced and when workers cannot increase their inclusive fitness by changing the proportion of female larvae raised as workers versus gynes. We can find the equilibrium for $f$ and $w$ by solving the equation system

$$
\begin{aligned}
& \left.\frac{\partial V_{Q}}{\partial f}\right|_{f=F=f^{*}}=0, \\
& \left.\frac{\partial V_{W}}{\partial w}\right|_{w=W=w^{*}}=0,
\end{aligned}
$$

while substituting the corresponding relatedness values. Under monandry and monogyny, the equilibrium values are $f^{*}=0.72$ and $w^{*}=0.51$, corresponding to a proportional investment of 0.36 in workers, 0.35 in gynes, and 0.29 in males. This indicates that colony productivity is suboptimal because the proportional investment in workers is $<0.42$. Sex allocation is intermediate between the queen and worker optima, the female-to-male investment ratio being at $1.2: 1$. (Note again that the numerical values should only be considered as indicative of the qualitative but not quantitative change in resource allocation.)

The ES values of $f$ and $w$ under polyandry or polygyny can be calculated by substituting the corresponding relatedness values in equation (2). Polyandry reduces the relatedness among female offspring of the queen (workers and gynes), whereas worker-male relatedness remains constant. Hence, relatedness asymmetry decreases as queen mating frequency increases and workers benefit less from biasing sex allocation toward females. Therefore, multiple mating reduces conflict between queens and workers over sex allocation, and ES resource allocation tends toward the values obtained under complete queen control (fig. $2 \mathrm{~A}$; calculations assume even paternity of the queen's mates). Concurrent with this tendency, higher queen mating frequencies result in an increase in the proportional invest-

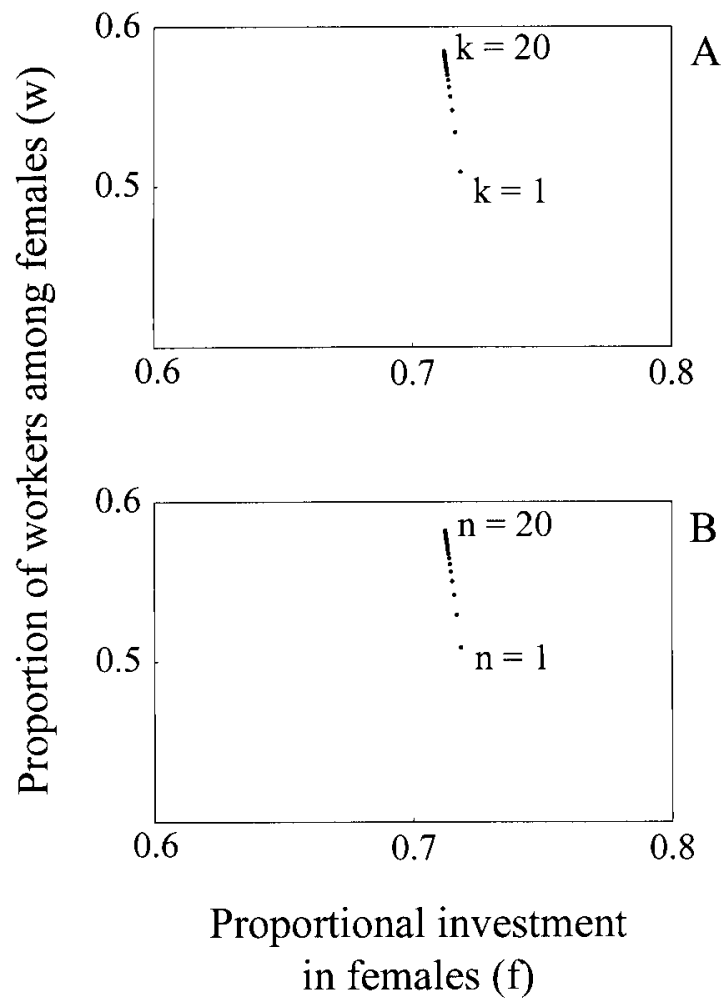

Figure 2: Evolutionary equilibria of proportional investment in females $(f)$, and proportion of workers among females $(w)$, as a function of $(A)$ queen mating frequency $(k$; assuming monogyny and equal paternity among males) and $(B)$ the number of nestmate queens ( $n$; assuming full sister queens $\left[g_{\mathrm{QQ}}=0.75\right]$, single mating, and equal contribution of queens to the production of workers, males, and gynes). Here, $f$ is assumed to be under queen control, whereas $w$ is controlled by the workers. 
ment in workers which asymptotically approaches the optimal value of 0.42 (fig. $3 A$ ).

Increased levels of polygyny also lead to a smaller female-biased sex allocation and to an increase in the relative investment in workers (figs. $2 \mathrm{~B}, 3 \mathrm{~B}$; calculations assume equal contribution of queens to the production of workers, gynes, and males). The effect of increased queen number is stronger the more closely queens are related. If queens are unrelated, the ES sex allocation and the relative investment in workers are not affected by changes in queen number and remain the same as under monandry and monogyny. This is because relatedness asymmetry is unaffected by variation in queen number when queens are unrelated (Frank 1987).

\section{Larval Control of Caste Determination}

So far we have only considered the workers' and queens' interests. We will now investigate whether the interests of the developing larvae themselves diverge from those of workers and queens. Diverging interests of female larvae would be indicated by selection favoring larvae who escaped adult control of caste determination. Whether this is the case can be investigated with our model. Consider a mutant larva that controls its own caste determination and develops with probability $\omega$ into a worker and $(1-$ $\omega)$ into a gyne. Other larvae in the colony in which the mutant occurred become workers and gynes with probabilities $w$ and $(1-w)$, respectively, whereby $w$ is determined by either the queen or workers. Assume also, for simplicity, that the population investment patterns are the same as in this colony, that is, $W=w$ and $F=f$.

The inclusive fitness of the mutant larva $V_{M}$ is equal to the sum of its inclusive fitness if developing into a worker $\left(\Delta V_{W}\right)$ and the inclusive fitness if developing into a gyne $\left(\Delta V_{G}\right)$, weighted by the respective probabilities, $V_{M}=$ $\omega\left(\Delta V_{W}\right)+(1-\omega)\left(\Delta V_{G}\right)$. The inclusive fitness gained by worker development $\Delta V_{W}$ is equal to the number of additional males and gynes a colony would produce with the help of one additional worker multiplied by the larva's relatedness to these sexual females and males and their mating success. The increase in colony productivity can be calculated as follows: if $s(f, w)=b(f, w)(1-f w)$ describes the relative production of sexuals in the focal colony, the number of extra sexuals produced as a result of one additional worker is given by $\delta s / f \delta w$. This expression is the slope of sexual production on investment in workers $(f w)$. The division by $f$ is necessary because overall colony productivity varies between 0 and 1 , whereas the investment in workers $(f w)$ varies between 0 (for $w=0$ ) and $f$ (for $w=1$ ). Dividing by $f$ corrects for this difference in scale, and one obtains the number of gynes produced per worker. The direct fitness obtained by a larva developing
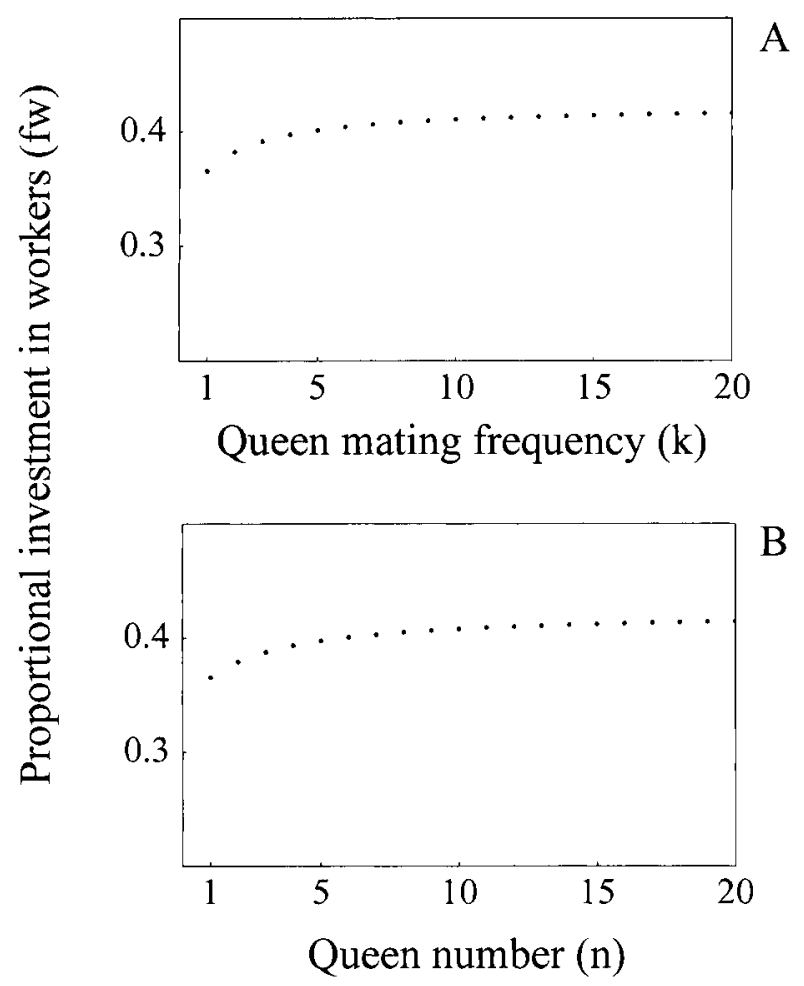

Figure 3: Evolutionary equilibria of proportional investment in workers $(f w)$ in the case where queens control the proportional investment in females $(f)$ and workers control the proportion of workers among females $(w)$, as a function of $(A)$ queen mating frequency ( $k$; assuming single-queen colonies and equal paternity among males), and $(B)$ the number of nestmate queens ( $n$; assuming full sister queens $\left[g_{Q Q}=0.75\right]$, single mating, and equal contribution of queens to the production of workers, males, and gynes).

into a gyne $\left(\Delta V_{G}\right)$ is simply equal to her expected mating success $(1 / F[1-W])$, its relatedness to itself being 1 . The overall mutant inclusive fitness can thus be written as

$$
\begin{aligned}
V_{M}= & \omega \frac{\delta s}{\delta w} \frac{1}{f}\left[\frac{f(1-w)}{(1-f w)} \frac{g_{f X} \nu_{f}}{F(1-W)}+\frac{(1-f)}{(1-f w)} \frac{g_{m X} \nu_{m}}{(1-F)}\right] \\
& +(1-\omega) \frac{1}{F(1-W)}
\end{aligned}
$$

The gradient of selection acting on $\omega$, the larva's probability of developing into a worker, is the partial derivative of $V_{M}$ with respect to $\omega$. This value depends on the colony and population investment pattern $(f, w)$. The selection pressure on $\omega$ for all $f$ and $w$ values in the range between the two extremes of complete worker and complete queen control is given in figure 4 . The selection gradient is always strongly negative, indicating that a single mutant larva 


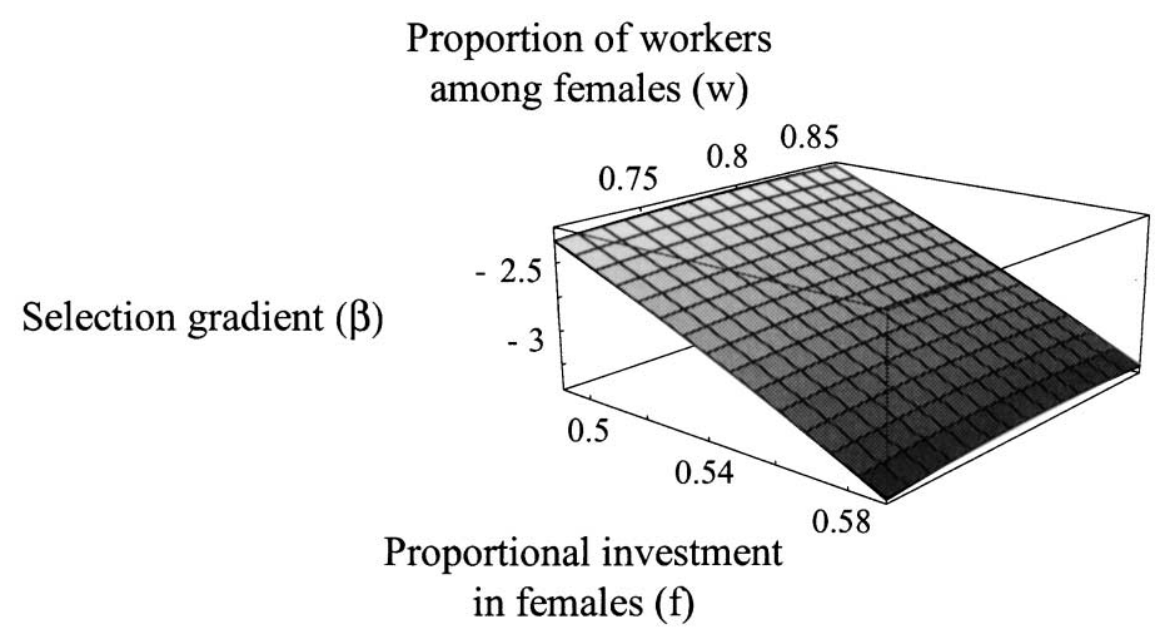

Figure 4: Selection differential $(\beta)$ on a mutant female larva's probability to develop into a worker $(\omega)$ as a function of egg sex ratio $(f)$ and proportion of female larvae raised as workers $(w)$.

always benefits to develop into a gyne, and this for all possible values of $f$ and $w$.

The question arises as to what will be the equilibrium resource allocation in the colony when all larvae are in control of their developmental fate. To investigate this, we need to determine the evolutionarily stable proportion of larvae $(w)$ developing into workers and the adaptive response of colony members (workers and queens) in terms of the proportion of females $f$ they would produce. To find the equilibrium, we have to maximize simultaneously the inclusive fitness of the larvae and the party controlling $f$. For larvae, we replace $\omega$ in equation (3) with $w$ (because at equilibrium all larvae adopt the same optimal strategy) and substitute the corresponding relatedness values. The resulting equation is derived with respect to $w$. For the party controlling $f$, we substitute the corresponding relatedness values in Equation 1 and derive with respect to $f$. The two derivatives are then set equal to 0 and solved for $f$ and $w$ at $f=F=f^{*}$ and $w=W=w^{*}$.

At equilibrium, the investment in workers is very low and the sex allocation strongly female biased. Under monandry, monogyny, and queen control of proportional investment in females, the equilibrium is at $f^{*}=0.74$ and $w^{*}=0.07$, corresponding to a proportional investment of 0.05 in workers, 0.69 in gynes, and 0.26 in males. If proportional investment in females is controlled by workers, the equilibrium is at $f^{*}=0.87$ and $w^{*}=0.09$, that is, a proportion of 0.08 of the resources is invested in workers, 0.80 in gynes, and 0.12 in males. The investment in workers will be even lower when queens are mated to several males (fig. $5 A, 5 C$ ) or if colonies contain several queens (fig. $5 B, 5 D$ ). The decrease in worker production associated with the higher levels of polygyny is more pronounced the less queens are related to each other.

Note that, as in previous analyses, the numerical values given here have no significance per se because they directly depend on the function $b(f, w)$ that was chosen. However, the qualitative results of the analyses are not affected by the particular function, as long as it is one with diminishing returns, and the important point is that larvae will always be in strong conflict with queens and workers over their developmental fate. Moreover, this conflict will be stronger and the proportion of larvae developing into workers lower, the higher the levels of polyandry and polygyny.

\section{Discussion}

Our model shows that potential conflict over investment in workers can arise in colonies of eusocial Hymenoptera. Although queens' and workers' inclusive fitness are maximal when the investment in workers is at the value that maximizes colony productivity of gynes (Bourke and Chan 1999, this study), this optimum will be reached only when a single party controls all decisions about resource allocation. However, our model shows that single-party control of resource allocation is not evolutionarily stable because sex ratio conflict results in strong selection on the other party to manipulate investment. Hence, the queen will benefit from forcing workers to produce a less femalebiased sex allocation by altering the primary sex ratio and limiting the number of eggs laid. Limitation of diploid eggs will, in turn, induce workers to direct a higher proportion of the female larvae into the gyne developmental 

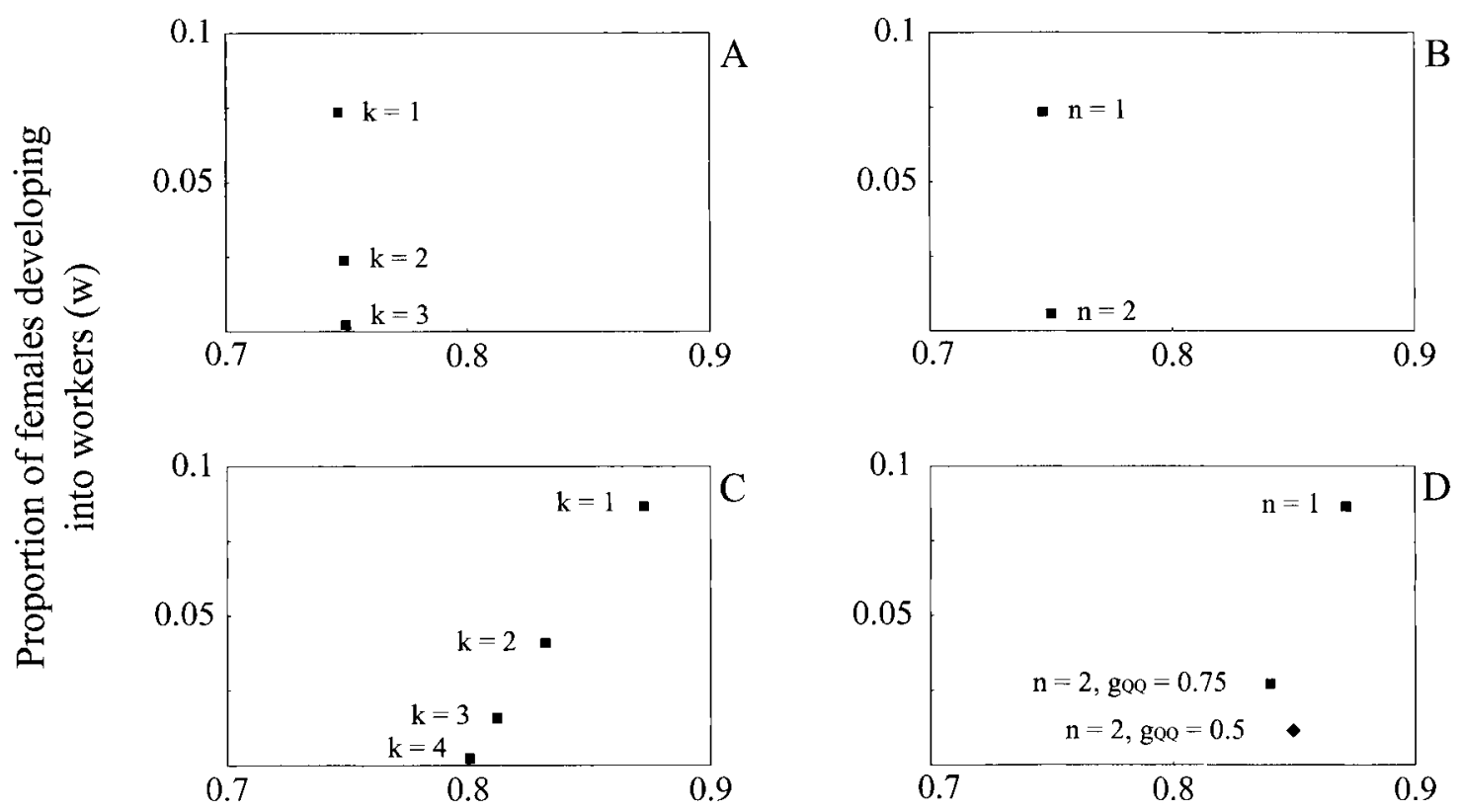

\section{Proportional investment in females (f)}

Figure 5: Evolutionary equilibria of the proportional investment in females $(f)$ and the proportion of female larvae developing into workers $(w)$ $(A)$ in function of queen mating frequency $(k)$ with queen control of proportional investment in females, $(B)$ in function of the number of nestmate queens $(n)$ with queen control of proportional investment in females, $(C)$ in function of queen mating frequency with worker control of proportional investment in females, and $(D)$ in function of the number of nestmate queens with worker control of proportional investment in females. Panels $A$ and $C$ assume monogyny and equal paternity among males, and panels $B$ and $D$ assume that queens are full sisters $\left(g_{Q Q}=0.75\right.$, squares $)$ or halfsisters ( $g_{\mathrm{QQ}}=0.5$, diamonds), single mating, and equal contribution of queens to the production of workers, males, and gynes.

pathway so as to achieve a more female-biased sex allocation. This tug-of-war between queens and workers results in an allocation of resources to workers that is lower than the optimal value for queens and workers, and a sex allocation intermediate between the queen and worker optima.

\section{Resolving Controversies among Earlier Work}

Our findings shed light on the causes of discrepancies between earlier theoretical studies on the existence of potential queen-worker conflict over colony growth. These studies had analyzed the problem from slightly differing angles and under varying assumptions. Two of them came to the conclusion that a conflict occurs (Pamilo 1991; Bourke and Ratnieks 1999), whereas another concluded that there is no conflict (Bourke and Chan 1999). Pamilo (1991) found that the queen favors a higher investment in colony growth than workers because queens value the survival of the colony more than workers (survival being higher with greater investment in workers). Workers, on the other hand, would benefit more than queens from investing in gyne production because they are more closely related to sexual females than are queens. Pamilo's explanation is surprising because his model did not include competition among colonies. Moreover, both queens and workers should benefit most from queen-derived sexual offspring compared to less related second-generation offspring (Bourke and Chan 1999). Similarly, Bourke and Ratnieks (1999) suggested that queen and workers should be in conflict over caste determination of individual female larvae. In their analysis the authors assumed that perworker productivity declines with increased colony size, and they graphically determined at which colony size each party switches preference from raising larvae as workers to raising them as gynes. Workers were found to favor a switch to gyne production at a smaller colony size than the queen. This is because workers are more closely related to the larvae, and thus the fitness gained from producing sexual sisters outweighs the benefits of increased overall production.

A detailed analysis of these two studies provides an explanation of why they predict a queen-worker conflict. To derive the evolutionarily stable strategy, Pamilo (1991, eq. 
[13]) compared for each party the inclusive fitness gained from investing into workers versus that gained through gynes. This is exactly the trade-off that occurs when diploid eggs are limited, in which case workers have to decrease the investment in workers to bias sex allocation toward more females. The same is true with Bourke and Ratnieks's (1999) analysis, because they considered the specific option to direct a female larva into the gyne versus worker developmental pathway. Hence, the different queen and worker optima stem from the decision ultimately affecting colony sex allocation. By contrast, Bourke and Chan's (1999) model assumes a trade-off between worker production versus colony productivity of sexuals (males and females) without assuming any effect of this trade-off on sex allocation. Our model shows that this is what occurs when a single party has full control over colony sex allocation, a situation that is indeed assumed in Bourke and Chan's (1999) model. As a consequence, Bourke and Chan (1999) found that queens and workers agree on the amount of resources to be directed to worker production.

\section{Larval Self-Determination}

Our model shows that female larvae and adult colony members are generally in strong conflict over caste determination, confirming and generalizing earlier analyses (Nonacs and Tobin 1992; Bourke and Ratnieks 1999). With monandry and monogyny, there is a wide range of conditions where female larvae benefit from becoming gynes, whereas queens and workers would prefer them to develop into workers. For more complex kin structure (polygyny and polyandry), the conflict is even stronger and the investment in workers lower, the predicted value being 0 under a wide range of conditions. However, the prediction of no larvae developing into workers has to be considered with caution because it is dependent on the specific relationship between per-worker productivity and colony size that was chosen. Moreover, our model does not include seasonal variation in the probability for gynes to successfully disperse and initiate new colonies. This may be an important factor with, for example, female larvae being more likely to develop into workers when the expected chances of successful dispersal are low.

The demonstration of a strong difference in interest between female larvae and adult individuals raises the question of who is in control of caste determination. In many species, caste determination is influenced by the amount and type of food provided to larvae (Wheeler 1986), suggesting that workers, which are usually the individuals that feed the larvae, should have a strong influence on caste determination. However, there are several means by which female larvae may gain some control over their developmental fate. First, in some species, larvae probably can influence the amount of resource they obtain and hence increase their probability of developing into a gyne (Bourke and Ratnieks 1999). For example, in pocketmaking bumblebees, larvae feed autonomously in communal mass-provisioned cells (Michener 1974; Bourke and Ratnieks 1999). Strong competition between larvae seems indeed to occur whereby larvae occupying a central position in the communal cell monopolize food and develop more frequently into gynes than larvae in the periphery (Michener 1974). Furthermore, workers of the pocketmaking bumblebees are more variable in size than workers of pollen-storer species, which rear larvae in individual cells continuously provisioned by workers (Michener 1974). The greater variance could stem from larvae's attempts to acquire more resources in order to trigger gyne development.

An alternative route to gyne development is to decrease the caste threshold (the size at which gyne development is triggered; Wheeler 1986), thus allowing development into a gyne with fewer resources than normally required. A reduction in gyne size and caste threshold has apparently evolved in parasitic ants (Nonacs and Tobin 1992; Aron et al. 1999). In these species, female larvae are under strong selection to develop into gynes because the worker tasks are performed by the host workers and worker development therefore offers only a relatively small fitness gain for parasite larvae. Reduction of queen size has also been documented in several nonparasitic Hymenoptera (Michener 1974; Brian 1983). Queens of reduced size (microgynes) occur, for example, in the bee Trigona julianii (Juliani 1967, cited in Michener 1974). It is interesting that, in this species, queens are usually produced in special cells but microgynes develop from worker cells (Michener 1974). This suggests that reduced size is the only way for a female to develop into a gyne when in a worker cell. Microgynes have also been found in several ants. However, it is yet unclear whether the smaller size of these microgynes has evolved as part of a selfish strategy to increase the probability of developing into a gyne or is the result of alternative reproductive strategies (Bourke and Franks 1995).

Finally, when a larva cannot modify the rate of food intake nor reduce size and caste threshold, another option is to increase its developmental time. However, it is unclear whether workers may counteract such selfish larval strategies, for example, by reducing the amount of food provided to larvae or being aggressive toward them. Workers of some Myrmica species have for instance been reported to provide less food to overwintering than to spring larvae (Brian 1983). Aggression toward larvae developing into gynes has been reported in several ant species (Fletcher 1986; Vargo and Passera 1991). The differential treatment 
of gyne-inclined larvae by workers may represent means to incite larvae to develop into workers.

\section{Identity of the Controlling Parties}

Our model makes predictions that may allow us to test which party controls reproductive decisions in the colony. It predicts that the relative investment in workers, first, should decrease with increased levels of polygyny and polyandry if larvae can influence their developmental fate, second, not be affected by the levels of polygyny and polyandry if either workers or queens are in full control of resource allocation, and third, increase with increased levels of polygyny and polyandry if workers and queens share control over resource allocation. These predictions can be tested by comparing the size of colonies varying in their kin structure because differences in the relative investment in workers should translate in size differences between mature colonies. Evidence for a lack of a relationship between colony size and kin structure comes from two ant species, Formica truncorum (Sundström and Ratnieks 1998) and Formica exsecta (Sundström et al. 1996). In both species, queens can mate singly or multiply, but colony size is apparently not associated with queen mating frequency. It is interesting that two lines of evidence suggest that workers may indeed have an important influence on resource allocation in these two species. First, the population-wide sex-investment ratio is very close to the workers' optimum (Sundström 1994; Sundström et al. 1996). Second, workers seem to have a significant influence on reproductive decisions at the colony level because colonies produce the sex to which workers are more related compared to the population average, as predicted by split sex ratio theory (Boomsma and Grafen 1990, 1991). That is, colonies produce mostly females when the queen is singly mated and males when the queen is multiply mated (Sundström 1994; Sundström et al. 1996). Workers seem to manipulate sex allocation to their advantage by selectively eliminating males in colonies headed by singly mated queens (Sundström et al. 1996). It remains to be investigated whether sex ratio manipulation also involves the differential allocation of female brood to sexual and worker development.

In the recent literature, queen control over sex allocation is usually indirectly inferred from an even sex allocation (e.g., Helms 1999). However, only rarely are attempts made to investigate empirically the proximate mechanisms of queen control that would present an alternative to pheromonal queen control (see Helms et al. 2000 for an exception). The lack of empirical tests is surprising given that several authors have proposed the idea that queens may prevent workers from biasing sex allocation toward females by limiting the number of diploid eggs laid (Bul- mer and Taylor 1981; Ratnieks and Reeve 1992; this study). Furthermore, the ability of queens to equilibrate the sex investment ratio by limiting diploid eggs is predicted to be lower in species with well-marked gyne-worker dimorphism (Bulmer and Taylor 1981) because, in species with costly gynes, even a low number of eggs raised as gynes represents a high investment in sexual females. Thus, a higher investment in gynes does not come at the cost of a significant decrease in colony productivity as a result of lower worker production.

The predicted association between gyne-worker dimorphism and queen's ability to manipulate colony sex allocation is intriguing because it may provide an explanation for the observed positive correlation between sexual dimorphism and female bias across ant species. Boomsma (1989) first noted the existence of this association and suggested that it might be due to a methodological artifact with female bias in sex investment ratio tending to be increasingly overestimated as sexual dimorphism increases (see also Boomsma et al. 1995). However, the association between sexual dimorphism and female bias is also predicted if queens limit the number of female eggs because sexual dimorphism tends to be positively correlated with gyne-worker dimorphism in ants (L. Keller, unpublished data). Hence, queens should have less control over sex allocation and it should be more female biased in species with greater sexual dimorphism. Experiments are needed to determine whether queen-worker conflict over sex allocation indeed leads to limitation of female eggs and whether the occurrence of egg limitation is negatively correlated with gyne-worker and gyne-male dimorphism. If such a negative correlation exists, one would expect a greater ability of workers to control resource allocation in species with a greater degree of sexual and gyne-worker dimorphism. This tendency toward complete worker control should translate into a greater investment in workers, and one would thus predict a positive relationship between gyne-worker dimorphism and colony size, as indeed is the case across species of social Hymenoptera (Bourke 1999). However, there are also other reasons to expect such an association (see Bourke 1999).

A situation similar to female egg limitation occurs when queens and males are successful in concealing the sex of the brood in order to resist the workers' manipulation of colony sex allocation (Nonacs and Carlin 1990; Nonacs 1993). If the deception is successful up to a developmental stage at which the fitness gain of biasing the sex allocation does not compensate for the loss of the energy already invested in rearing the males, the workers are forced to raise offspring in the sex ratio produced by the queen (Nonacs and Carlin 1990; Chapuisat et al. 1997). Under such conditions, the only reproductive decision possibly remaining under the control of the workers is the pro- 
portion of females developing into gynes versus workers, given that female caste can be influenced beyond the point where brood sex becomes apparent. There is limited evidence that sexual deception may occur in ants (Nonacs and Carlin 1990; Chapuisat et al. 1997), but well-designed experiments still need to be conducted to demonstrate unambiguously its occurrence.

\section{Conclusion}

This study explicitly delineates the conditions under which there is a conflict between queens, workers, and larvae over resource allocation to the production of workers, gynes, and males. It makes predictions about how variation in queen number and queen mating frequency should affect worker production depending on which party controls resource allocation. Our model also integrates key life-history parameters in sex allocation theory, which will hopefully stimulate more empirical research on the strategies pursued by queens, workers, and female brood to maximize their inclusive fitness. These studies would help to broaden our understanding of the factors shaping the life history of hymenopteran colonies and may provide explanations for many examples of observed sex allocation patterns that cannot be accounted for by the models of sex ratio theory available so far (Bourke and Franks 1995; Crozier and Pamilo 1996; Chapuisat and Keller 1999).

\section{Acknowledgments}

We would like to thank N. Perrin for very helpful discussions on the model and F. Balloux, A. Bourke, M. Chapuisat, D. Feener, J. Greeff, K. Helms, D. Hosken, P. Nonacs, P. Pamilo, J. Parker, and three anonymous referees for comments on the manuscript. This work was supported by grants from the Swiss National Science Foundation.

\section{Literature Cited}

Aron, S., L. Passera, and L. Keller. 1999. Evolution of social parasitism in ants: size of sexuals, sex ratio and mechanisms of caste determination. Proceedings of the Royal Society of London B, Biological Sciences 266:173-177.

Boomsma, J. J. 1989. Sex-investment ratios in ants: has female bias been systematically overestimated? American Naturalist 133:517-532.

Boomsma, J. J., and A. Grafen. 1990. Intraspecific variation in ant sex ratios and the Trivers-Hare hypothesis. Evolution 44:1026-1034. 1991. Colony-level sex ratio selection in the eusocial Hymenoptera. Journal of Evolutionary Biology 3: 383-407.

Boomsma, J. J., L. Keller, and M. G. Nielsen. 1995. A com- parative analysis of sex ratio investment parameters in ants. Functional Ecology 9:743-753.

Bourke, A. F. G. 1999. Colony size, social complexity and reproductive conflict in social insects. Journal of Evolutionary Biology 12:245-257.

Bourke, A. F. G., and G. L. Chan. 1999. Queen-worker conflict over sexual production and colony maintenance. American Naturalist 154:417-426.

Bourke, A. F. G., and N. R. Franks. 1995. Social evolution in ants. Princeton University Press, Princeton, N.J.

Bourke, A. F. G., and F. L. W. Ratnieks. 1999. Kin conflict over caste determination in social Hymenoptera. Behavioral Ecology and Sociobiology 46:287-197.

Brian, M. V. 1956. Group form and the cause of working inefficiency in the ant Myrmica rubra L. Physiological Zoology 29:173-194.

- 1980. Social control over sex and caste in bees, wasps and ants. Biological Reviews of the Cambridge Philosophical Society 55:379-415.

- 1983. Social insects: ecology and behavioural biology. Chapman \& Hall, London.

Bulmer, M., and P. D. Taylor. 1981. Worker-queen conflict and sex ratio theory in social Hymenoptera. Heredity 47:197-207.

Chapuisat, M., and L. Keller. 1999. Testing kin selection with sex allocation data in eusocial Hymenoptera. Heredity $82: 473-478$.

Chapuisat, M., L. Sundström, and L. Keller. 1997. Sexratio regulation: the economics of fratricide in ants. Proceedings of the Royal Society of London B, Biological Sciences 264:1255-1260.

Charnov, E. L. 1978. Sex-ratio selection in eusocial Hymenoptera. American Naturalist 112:317-326.

Clark, A. B. 1978. Sex ratio and local resource competition in a prosimian primate. Science (Washington, D.C.) 201: 163-165.

Crozier, R. H., and P. Pamilo. 1996. Evolution of social insect colonies: sex allocation and kin-selection. Oxford University Press, Oxford.

Fletcher, D. J. C. 1986. Triple action of queen pheromones in the regulation of reproduction in fire ant (Solenopsis invicta) colonies. Pages 305-316 in M. Porchet, J.-C. Andries, and A. Dhaiant, eds. Advances in invertebrate reproduction. Vol. 4. Elsevier, Amsterdam.

Fletcher, D. J. C., and K. G. Ross. 1985. Regulation of reproduction in eusocial Hymenoptera. Annual Review of Entomology 30:319-343.

Frank, S. A. 1987. Variable sex ratio among colonies of ants. Behavioral Ecology and Sociobiology 20:195-201.

Hamilton, W. D. 1964. The genetical evolution of social behavior. II. Journal of Theoretical Biology 7:17-52.

- 1967. Extraordinary sex ratios. Science (Washington, D.C.) 156:477-488. 
Helms, K. R. 1999. Colony sex ratios, conflict between queens and workers, and apparent queen control in the ant Pheidole desertorum. Evolution 53:1470-1478.

Helms, K. R., J. H. Fewell, and S. W. Rissing. 2000. Sex ratio determination by queens and workers in the ant Pheidole desertorum. Animal Behaviour 59:523-527.

Hölldobler, B., and S. Bartz. 1985. Sociobiology of reproduction in ants. Pages 237-257 in B. Hölldobler and M. Lindauer, eds. Experimental behavioral ecology and sociobiology. G. Fischer, Stuttgart.

Juliani, L. 1967. A descriçao do ninho e alguns dados biológicos sôbre a abelha Plebeia julianii Moure 1962 (Hymenoptera, Apoidea). Revista Brasileira de Entomologia (São Paulo) 12:31-58.

Keller, L., and M. Chapuisat. 1999. Cooperation among selfish individuals in insect societies. BioScience 49: 899-909.

Keller, L., and P. Nonacs. 1993. The role of queen pheromones in colonies of social insects: queen control or queen signal? Animal Behaviour 45:787-794.

Keller, L., and H. K. Reeve. 1999. Dynamics of conflicts within insect societies. Pages 153-175 in L. Keller, ed. Levels of selection in evolution. Princeton University Press, Princeton, N.J.

Lande, R., and S. J. Arnold. 1983. The measurement of selection on correlated characters. Evolution 37: 1210-1226.

Michener, C. D. 1974. The social behavior of bees: a comparative study. Harvard University Press, Cambridge, Mass.

Nonacs, P. 1986. Ant reproductive strategies and sex allocation theory. Quarterly Review of Biology 61:1-21.

- 1993. Male parentage and sexual deception in the social Hymenoptera. Pages 384-401 in D. L. Wrensch and M. Ebberts, eds. Evolution and diversity of sex ratio in insects and mites. Chapman \& Hall, New York.

Nonacs, P., and N. F. Carlin. 1990. When can ants discriminate the sex of brood? a new aspect of queenworker conflict. Proceedings of the National Academy of Sciences of the USA 87:9670-9673.

Nonacs, P., and J. Tobin. 1992. Selfish larvae: development and the evolution of parasitic behavior in the Hymenoptera. Evolution 46:1605-1620.

Ortius, D., and J. Heinze. 1999. Fertility signaling in queens of a North American ant. Behavioral Ecology and Sociobiology 45:151-159.

Pamilo, P. 1982. Genetic evolution of sex ratios in eusocial Hymenoptera: allele frequency simulations. American Naturalist 119:638-656.
1991. Evolution of colony characteristics in social insects. I. Sex allocation. American Naturalist 137: 83-107.

Passera, L. 1980. La ponte d'oeufs préorientés chez la fourmi Pheidole pallidula (Nyl.) (Hymenoptera: Formicidae). Insectes Sociaux 27:79-95.

Pettis, J. S., H. A. Higo, T. Pankiw, and M. L. Winston. 1997. Queen rearing suppression in the honey bee-evidence for a fecundity signal. Insectes Sociaux 44:311-322.

Ratnieks, F. L. W., and H. K. Reeve. 1992. Conflict in single-queen hymenopteran societies: the structure of conflict and processes that reduce conflict in advanced eusocial species. Journal of Theoretical Biology 158: 33-65.

Rosenheim, J. A., P. Nonacs, and M. Mangel. 1996. Sex ratios and multifaceted parental investment. American Naturalist 148:501-535.

Stearns, S. C. 1992. The evolution of life histories. Oxford University Press, Oxford.

Sundström, L. 1994. Sex ratio bias, relatedness asymmetry and queen mating frequency in ants. Nature (London) 367:266-268.

-1995. Sex allocation and colony maintenance in monogyne and polygyne colonies of Formica truncorum (Hymenoptera, Formicidae) — the impact of kinship and mating structure. American Naturalist 146:182-201.

Sundström, L., and F. L. W. Ratnieks. 1998. Sex ratio conflicts, mating frequency, and queen fitness in the ant Formica truncorum. Behavioral Ecology 9:116-121.

Sundström, L., M. Chapuisat, and L. Keller. 1996. Conditional manipulation of sex ratios by ant workers-a test of kin selection theory. Science (Washington, D.C.) 274:993-995.

Trivers, R. L., and H. Hare. 1976. Haplodiploidy and the evolution of the social insects. Science (Washington, D.C.) 191:249-263.

Tschinkel, W. R. 1993. Sociometry and sociogenesis of colonies of the fire ant Solenopsis invicta during one annual cycle. Ecological Monographs 63:427-457.

Vargo, E. L., and L. Passera. 1991. Pheromonal and behavioral queen control over the production of gynes in the Argentine ant Iridomyrmex humilis (Mayr). Behavioral Ecology and Sociobiology 28:161-169.

Wheeler, D. E. 1986. Developmental and physiological determinants of caste in social Hymenoptera: evolutionary implications. American Naturalist 128:13-34.

Associate Editor: Donald H. Feener, Jr. 